\title{
Local Approximations for Effective Scalar Field Equations of Motion
}

\author{
Arjun Berera,, , 田 Ian G. Moss,, , 团 and Rudnei O. Ramos 3 , 国 \\ ${ }^{1}$ School of Physics, University of Edinburgh, Edinburgh, EH9 3JZ, United Kingdom \\ ${ }^{2}$ School of Mathematics and Statistics, University of Newcastle Upon Tyne, NE1 7RU, United Kingdom \\ ${ }^{3}$ Departamento de Física Teórica, Universidade do Estado do Rio de Janeiro, 20550-013 Rio de Janeiro, RJ, Brazil
}

\begin{abstract}
Fluctuation and dissipation dynamics is examined at all temperature ranges for the general case of a background time evolving scalar field coupled to heavy intermediate quantum fields which in turn are coupled to light quantum fields. The evolution of the background field induces particle production from the light fields through the action of the intermediate catalyzing heavy fields. Such field configurations are generically present in most particle physics models, including Grand Unified and Supersymmetry theories, with application of this mechanism possible in inflation, heavy ion collision and phase transition dynamics. The effective evolution equation for the background field is obtained and a fluctuation-dissipation theorem is derived for this system. The effective evolution in general is nonlocal in time. Appropriate conditions are found for when these time nonlocal effects can be approximated by local terms. Here careful distinction is made between a local expansion and the special case of a derivative expansion to all orders, which requires analytic behavior of the evolution equation in Fourier space.
\end{abstract}

PACS numbers: $98.80 . \mathrm{Cq}, 11.10 . \mathrm{Wx}$

\section{INTRODUCTION}

Dissipation physics can play an important role in systems governed by quantum fields in subject areas as diverse as condensed matter to particle physics and early universe cosmology. Dissipation effects can have experimental consequences in many systems. In cosmology, inflation could occur concurrently with particle production due to dissipation from the scalar inflaton field in the context of the warm inflation scenario [1, 2], leading to observable signatures in density perturbations [3, 4]. In heavy ion collisions, the chiral symmetry QCD transition could be accompanied by dissipative effects [5]. More generally, any phase transition in a condensed matter, particle physics or cosmological system will be accompanied by dissipative effects during the dynamical evolution of the order parameter characterizing the phase transition [ [6].

The first principles origin of dissipation from quantum field theory has been a subject of interest for several decades. The typical system studied [7, 8, 9, 10, 11, 13, 14] is where a background scalar field is coupled to some variety of bosonic and fermionic light quantum fields, with the entire system starting off in some statistical state. The general outcome is the time evolution of the scalar field induces production of particles from the light quantum fields, creating dissipative and fluctuating effects. Such dynamics is of interest in particle physics, since this direct dissipative mechanism arises from very generic coupling schemes and also because such dynamical situations commonly present themselves in problems such as inflationary cosmology, heavy ion physics and in phase transitions in general.

In particle physics models there is a novel interaction configuration which also has been shown to lead to considerable dissipative effects, and that is the case where a background scalar field is coupled to heavy intermediate quantum fields which in turn are coupled to the light quantum fields [15, 16]. In this two-stage mechanism, the background scalar field indirectly induces particle production in the light fields through the intermediate heavy fields which in a sense help to catalyze the effect. Dissipation from such field configurations in systems near thermal equilibrium has been shown to arise [17] and application of these effects to inflation have been shown to have significant importance [18].

A full understanding of this mechanism requires understanding not only the dissipative effects but also the fluctuations created and the relation between the two in the context of a fluctuation-dissipation theorem. This so far has not been done for this two-stage mechanism and will be one of the results of this paper.

To arrive at this result, what will be required is to obtain the effective evolution equation for the background scalar field once all other fields to which it is coupled are integrated out. The resulting effective evolution equation, which

\footnotetext{
*Electronic address: ab@ph.ed.ac.uk

†Electronic address: ian.moss@ncl.ac.uk

‡Electronic address: rudnei@uerj.br
} 
will be derived using the Schwinger closed-time formalism [19], in general will have time nonlocal terms. The second interest of this paper is in determining when such nonlocal terms can be well approximated by local terms. In general this requires a careful examination of the different time scales involved in the dynamics of the system in study to determine the importance of nonlocal effects. For instance, when the characteristic time scale of the nonlocal (or memory) terms in the effective equation of motion is close to the typical reaction time of the system, it has been shown that large effects on the dynamics can arise as a consequence of the nonlocal terms as compared to the results coming from a local approximation for the equations [20]. In this paper we adopted the general approach of directly analyzing the nonlocal terms and then determining the relevant time scale for when we can approximate them as local forms.

In practical application of these types of equations, there is an immense saving of effort as well as much more transparent understanding of the physics from a local equation as opposed to a nonlocal one, since the former can generally be analyzed with much less numerical treatment than the latter. Thus it is a very important question when and how accurately the generally nonlocal effective equations can be approximated by a local form. One very special type of local approximation is the derivative expansion 17, 11, 13, 14, 21, 22]. This is just a Taylor expansion of the nonlocal terms about some time point. Such an expansion therefore requires analytic behavior of the relevant nonlocal terms. However that may not arise in all circumstances, and for example even simple logarithmic corrections would make the derivative expansion invalid. Nevertheless, a careful distinction must be made between localized behavior as opposed to localized analytic behavior. Even in the general circumstance where the behavior is not analytic, the nonlocal terms may still be fairly peaked functions that can be well approximated as local. The nonlocal terms in the effective evolution equation we derive for this two-stage mechanism will be examined for when they can be approximated as localized. Although in this paper we carry out this investigation in the context of the two-stage mechanism, most of our significant results here would also apply to the direct dissipative mechanism (for a thorough discussion about the two-stage decay mechanism contrasted to the direct one, see e.g. Ref. [15, 16]).

We assume throughout that the system is enclosed by an environment which is initially close to thermal equilibrium and remains in that state due to the existence of a relatively short interaction timescale in the various components contributing to the environment. In this respect we have a different situation than the one considered in the original work on the two stage mechanism [15, 16] and more recent numerical work [23]. In fact, we find that the local dissipative effects cease in the limit that the temperature $T \rightarrow 0$, as one might expect and in agreement with [17, 23].

This paper is organized as follows. In Sec. II we give the Lagrangian density model used to study the two-stage decay mechanism and the respective effective equation of motion for a background scalar field that exhibits both the characteristic dissipation and fluctuation (stochastic) like terms. In Sec. III we derive the various generalized fluctuation-dissipation relations for the model under study and show the generality of these relations. In Sec. IV we define the local limit for the noise and dissipation terms and then we provide a thorough study both analytically and numerical to support localization in time for the noise and dissipation kernels appearing in the effective equation of motion given in Sec. II. Finally, our concluding remarks are given in Sec. V.

\section{THE MODEL}

We consider the following model of a scalar field $\Phi$ interacting indirectly through a catalyst field $\chi$ to a scalar field $\sigma$, which we can think of as our heat bath. The field $\Phi$ has no direct coupling terms to the field $\sigma$. The Lagrangian density for this model is the following

$$
\begin{aligned}
\mathcal{L}[\Phi, \chi, \sigma] & =\frac{1}{2} \partial_{\mu} \Phi \partial_{\nu} \Phi-\frac{m_{\phi}^{2}}{2} \Phi^{2}-\frac{\lambda}{4 !} \Phi^{4} \\
& +\frac{1}{2} \partial_{\mu} \chi \partial_{\nu} \chi-\frac{m_{\chi}^{2}}{2} \chi^{2}-\frac{f}{4 !} \chi^{4}-\frac{g^{2}}{2} \Phi^{2} \chi^{2} \\
& +\frac{1}{2} \partial_{\mu} \sigma \partial_{\nu} \sigma-\frac{m_{\sigma}^{2}}{2} \sigma^{2}-h \mathcal{M} \chi \sigma^{2}
\end{aligned}
$$

where $\mathcal{M}$ is a parameter with mass dimension added in order to make the coupling constant $h$ dimensionless.

We consider $\Phi(x)=\varphi_{c}(x)+\phi(x)$ where $\varphi_{c}(x)$ is a background like component of the scalar field, or vacuum expectation value of $\Phi$, and $\phi(x)$ are the quantum fluctuations around this background field. The nonequilibrium equation of motion for $\varphi_{c}$ can be derived using for example the functional closed time path (CTP) formulation of Schwinger-Keldysh [19]. In the CTP formalism the time integration is along a contour $c$ from $-\infty$ to $+\infty$ and then back to $-\infty$. For reviews please see, for example, Refs. 24, 25, 26]. The detailed derivation of the effective equation of motion (EOM) for the field $\varphi_{c}$ was explained in many previous papers (see e.g. [11, 15, 16, 27]) and therefore we 
will only give here the final result for it. The final result is expressed in terms of effective propagators (that already include the effects of the bath fields). In the CTP formalism these field propagators, e.g. for $\phi$, are given by [1] (which also apply to any of the other boson fields):

$$
\begin{aligned}
& G_{\phi}^{++}\left(x, x^{\prime}\right)=i\left\langle T_{+} \phi(x) \phi\left(x^{\prime}\right)\right\rangle, \\
& G_{\phi}^{--}\left(x, x^{\prime}\right)=i\left\langle T_{-} \phi(x) \phi\left(x^{\prime}\right)\right\rangle, \\
& G_{\phi}^{+-}\left(x, x^{\prime}\right)=i\left\langle\phi\left(x^{\prime}\right) \phi(x)\right\rangle, \\
& G_{\phi}^{-+}\left(x, x^{\prime}\right)=i\left\langle\phi(x) \phi\left(x^{\prime}\right)\right\rangle,
\end{aligned}
$$

where $T_{+}$and $T_{-}$indicate chronological and anti-chronological ordering, respectively. $G_{\phi}^{++}$is the usual Feynman propagator. The other three propagators come as a consequence of the time contour and are considered as auxiliary (unphysical) propagators. From (2.2) it also follows that the boson field propagators can also be expressed as

$$
\begin{aligned}
& G_{\phi}^{++}\left(x, x^{\prime}\right)=G_{\phi}^{>}\left(x, x^{\prime}\right) \theta\left(t-t^{\prime}\right)+G_{\phi}^{<}\left(x, x^{\prime}\right) \theta\left(t^{\prime}-t\right), \\
& G_{\phi}^{--}\left(x, x^{\prime}\right)=G_{\phi}^{>}\left(x, x^{\prime}\right) \theta\left(t^{\prime}-t\right)+G_{\phi}^{<}\left(x, x^{\prime}\right) \theta\left(t-t^{\prime}\right), \\
& G_{\phi}^{+-}\left(x, x^{\prime}\right)=G_{\phi}^{<}\left(x, x^{\prime}\right), \\
& G_{\phi}^{-+}\left(x, x^{\prime}\right)=G_{\phi}^{>}\left(x, x^{\prime}\right) .
\end{aligned}
$$

In deriving the effective EOM for $\varphi_{c}$ we first integrate over the $\sigma$ field. In this first step the Green's functions for the fields (in this case for the $\chi$ field in leading order) coupled to the fermions get dressed by $\sigma$ field corrections. Subsequently functional integration over the other fields (in our case the $\chi$ and $\phi$ field fluctuations) is performed. In this process, we can express the boson Green's functions entering in the $\varphi_{c}$ effective EOM in the generic form

$$
\left[\frac{\partial^{2}}{\partial t^{2}}-\nabla^{2}+M^{2}\right] G\left(x, x^{\prime}\right)+\int d^{4} z \Sigma(x, z) G\left(z, x^{\prime}\right)=\delta\left(x, x^{\prime}\right),
$$

where $G\left(x, x^{\prime}\right)$ and $\Sigma\left(x, x^{\prime}\right)$ are the (matrix) propagator and self-energy, respectively. The mass term $M^{2}$ in the above equation is the one appropriate the the respective field.

In terms of a loop expansion for the fluctuation field $\phi$ and $\chi$ and the generic expressions for the field propagators given above, the effective equation of motion that emerges for $\varphi_{c}$ is of the form [11, 15, 16, 27]

$$
\begin{aligned}
{[} & \left.+M_{\phi}^{2}\right] \varphi_{c}(x)+\frac{\lambda}{3 !} \varphi_{c}^{3}(x) \\
& +\varphi_{c}(x) \int d^{4} x^{\prime} \varphi_{c}^{2}\left(x^{\prime}\right)\left\{\frac{\lambda^{2}}{2} \operatorname{Im}\left[G_{\phi}^{++}\left(x, x^{\prime}\right)\right]^{2}+2 g^{4} \operatorname{Im}\left[G_{\chi_{j}}^{++}\left(x, x^{\prime}\right)\right]^{2}\right\} \theta\left(t-t^{\prime}\right) \\
& +\int d^{4} x^{\prime} \varphi_{c}\left(x^{\prime}\right)\left\{\frac{\lambda^{2}}{3} \operatorname{Im}\left[G_{\phi}^{++}\left(x, x^{\prime}\right)\right]^{3}+4 g^{4} \operatorname{Im}\left[G_{\chi}^{++}\left(x, x^{\prime}\right) G_{\phi}^{++}\left(x, x^{\prime}\right) G_{\chi}^{++}\left(x, x^{\prime}\right)\right]\right\} \theta\left(t-t^{\prime}\right) \\
& =\varphi_{c}(x) \xi_{1}(x)+\xi_{2}(x),
\end{aligned}
$$

where $M_{\phi}$ denotes the renormalized effective mass for $\phi$ that includes all local contributions. The nonlocal contributions can be associated to dissipation and noise terms. For instance, the third and forth terms in Eq. (2.5) are related to dissipation terms. The source terms $\xi_{1}$ and $\xi_{2}$ are stochastic (noise) fields associated with the imaginary terms in the effective action coming from the real-time evaluation of the perturbative loop diagrams leading to Eq (2.5). They have two-point correlation functions given by [11]

$$
\left\langle\xi_{1}(x) \xi_{1}\left(x^{\prime}\right)\right\rangle=\frac{\lambda^{2}}{2} \operatorname{Re}\left[G_{\phi}^{++}\left(x, x^{\prime}\right)\right]^{2}+2 g^{4} \operatorname{Re}\left[G_{\chi}^{++}\left(x, x^{\prime}\right)\right]^{2}
$$

and

$$
\left\langle\xi_{2}(x) \xi_{2}\left(x^{\prime}\right)\right\rangle=\frac{\lambda^{2}}{6} \operatorname{Re}\left[G_{\phi}^{++}\left(x, x^{\prime}\right)\right]^{3}+2 g^{4} \operatorname{Re}\left[G_{\chi}^{++}\left(x, x^{\prime}\right)^{2} G_{\phi}^{++}\left(x, x^{\prime}\right)\right]
$$


and are colored (space-time dependent) in general and Gaussian distributed, with probability distributions given by ( $N_{1}$ and $N_{2}$ are appropriate normalization constants)

$$
P\left[\xi_{1}\right]=N_{1}^{-1} \exp \left\{-\frac{1}{2} \int d^{4} x d^{4} x^{\prime} \xi_{1}(x)\left[\frac{\lambda^{2}}{2} \operatorname{Re}\left[G_{\phi}^{++}\left(x, x^{\prime}\right)\right]^{2}+2 \sum_{j} g_{j}^{4} \operatorname{Re}\left[G_{\chi_{j}}^{++}\left(x, x^{\prime}\right)\right]^{2}\right]^{-1} \xi_{1}\left(x^{\prime}\right)\right\}
$$

and

$$
P\left[\xi_{2}\right]=N_{2}^{-1} \exp \left\{-\frac{1}{2} \int d^{4} x d^{4} x^{\prime} \xi_{2}(x)\left[\frac{\lambda^{2}}{6} \operatorname{Re}\left[G_{\phi}^{++}\left(x, x^{\prime}\right)\right]^{3}+2 g^{4} \operatorname{Re}\left[G_{\chi}^{++}\left(x, x^{\prime}\right)^{2} G_{\phi}^{++}\left(x, x^{\prime}\right)\right]\right]^{-1} \xi_{2}\left(x^{\prime}\right)\right\} .
$$

Both dissipation and noise kernel are related to generalized fluctuation-dissipation relations, as we show next.

\section{FLUCTUATION-DISSIPATION RELATIONS}

A generalized fluctuation-dissipation relation between the nonlocal kernels in Eq. (2.5) and the stochastic fields correlation functions were derived in Ref. [11], while explicit expressions derived from the dissipation and noise kernels appearing in (2.5) were obtained by Yokoyama in Ref. [27]. General expressions that relate both kernels can also easily be derived in the following way. Lets first define dissipative kernels, $\mathcal{D}_{i}$, as given by

$$
\mathcal{C}_{i}\left(\mathbf{x}-\mathbf{x}^{\prime}, t-t^{\prime}\right)=-\frac{\partial}{\partial t^{\prime}} \mathcal{D}_{i}\left(\mathbf{x}-\mathbf{x}^{\prime}, t-t^{\prime}\right)
$$

where the $\mathcal{C}_{i=1,2}$ are defined by

$$
\mathcal{C}_{1}\left(\mathbf{x}-\mathbf{x}^{\prime}, t-t^{\prime}\right)=\lambda^{2} \operatorname{Im}\left[G_{\phi}^{++}\left(x, x^{\prime}\right)\right]^{2} \operatorname{sgn}\left(t-t^{\prime}\right)+4 g^{4} \operatorname{Im}\left[G_{\chi_{j}}^{++}\left(x, x^{\prime}\right)\right]^{2} \operatorname{sgn}\left(t-t^{\prime}\right),
$$

and

$$
\mathcal{C}_{2}\left(\mathbf{x}-\mathbf{x}^{\prime}, t-t^{\prime}\right)=\frac{\lambda^{2}}{3} \operatorname{Im}\left[G_{\phi}^{++}\left(x, x^{\prime}\right)\right]^{3} \operatorname{sgn}\left(t-t^{\prime}\right)+4 g^{4} \operatorname{Im}\left[G_{\chi}^{++}\left(x, x^{\prime}\right) G_{\phi}^{++}\left(x, x^{\prime}\right) G_{\chi}^{++}\left(x, x^{\prime}\right)\right] \operatorname{sgn}\left(t-t^{\prime}\right)
$$

where $\operatorname{sgn}\left(t-t^{\prime}\right)=\theta\left(t-t^{\prime}\right)-\theta\left(t^{\prime}-t\right)$ is the sign function (note that only the part for $t>t^{\prime}$ of these functions enters in the equation of motion Eq. (2.5) ). The extra factor 2 in the definition of Eq. (3.2) is for convention purposes, such that both $\mathcal{C}_{i=1,2}$ will satisfy the same fluctuation-dissipation relation. Using Eqs. (3.2) and (3.3) in (2.5) it follows that

$$
\begin{aligned}
{[} & \left.+M_{\phi}^{2}\right] \varphi_{c}(x)+\frac{\lambda}{3 !} \varphi_{c}^{3}(x) \\
& -\frac{\varphi_{c}(x)}{2} \int d^{3} x^{\prime} \varphi_{c}^{2}\left(\mathbf{x}^{\prime}, t\right) \mathcal{D}_{1}\left(\mathbf{x}-\mathbf{x}^{\prime}, 0\right)-\int d^{3} x^{\prime} \varphi_{c}\left(\mathbf{x}^{\prime}, t\right) \mathcal{D}_{2}\left(\mathbf{x}-\mathbf{x}^{\prime}, 0\right) \\
& +\varphi_{c}(x) \int d^{3} x^{\prime} \int_{-\infty}^{t} d t^{\prime} \varphi_{c}\left(\mathbf{x}^{\prime}, t^{\prime}\right) \dot{\varphi}_{c}\left(\mathbf{x}^{\prime}, t^{\prime}\right) \mathcal{D}_{1}\left(\mathbf{x}-\mathbf{x}^{\prime}, t-t^{\prime}\right)+\int d^{3} x^{\prime} \int_{-\infty}^{t} d t^{\prime} \dot{\varphi}_{c}\left(\mathbf{x}^{\prime}, t^{\prime}\right) \mathcal{D}_{2}\left(\mathbf{x}-\mathbf{x}^{\prime}, t-t^{\prime}\right) \\
& =\varphi_{c}(x) \xi_{1}(x)+\xi_{2}(x) .
\end{aligned}
$$

The third and forth terms in Eq. (3.4) can be shown to correspond, in the homogeneous approximation for the field $\varphi_{c}$, to the one-loop $\Phi$ vertex correction and the two-loop setting sun like mass corrections, respectively, for the model (2.1). Its is clear now from (3.4) that $\mathcal{D}_{i}$ are dissipative like terms. That they satisfy generalized fluctuationdissipation relations with the noise kernels defined by Eqs. (2.6) and (2.7) can be demonstrated when space-time Fourier transforming both kernels. We can write the Fourier transformed kernel $\mathcal{F}\left[\mathcal{C}_{i}\left(\mathbf{x}-\mathbf{x}^{\prime}, t-t^{\prime}\right)\right]=\tilde{\mathcal{C}}_{i}(\mathbf{p}, \omega)$, where the Fourier transform $\mathcal{F}[\cdots]$ is defined by 


$$
\mathcal{F}[\cdots]=\int d^{3} x d t[\cdots] e^{-i \mathbf{p} \cdot \mathbf{x}+i \omega t} .
$$

Note that, from the Eqs. (3.2) and (3.3), the kernels $\mathcal{C}_{i}$ are anti-symmetrical in the time. It then follows that their time Fourier transforms are purely imaginary. Thus, we can write [27]

$$
\tilde{\mathcal{C}}_{i}(\mathbf{p}, \omega)=-2 i \omega \tilde{\Gamma}_{i}(\mathbf{p}, \omega),
$$

where $\tilde{\Gamma}_{i}(\mathbf{p}, \omega)$ is a real quantity. The transformed dissipation kernels become

$$
\tilde{\mathcal{D}}_{i}(\mathbf{p}, \omega)=2 \tilde{\Gamma}_{i}(\mathbf{p}, \omega) .
$$

Similarly, for the noise terms we introduce the kernels $\mathcal{N}_{i}\left(\mathbf{x}-\mathbf{x}^{\prime}, t-t^{\prime}\right)$, where from Eqs. (2.6) and (2.7), they are defined by

$$
\mathcal{N}_{1}\left(\mathbf{x}-\mathbf{x}^{\prime}, t-t^{\prime}\right)=\frac{\lambda^{2}}{2} \operatorname{Re}\left[G_{\phi}^{++}\left(x, x^{\prime}\right)\right]^{2}+2 g^{4} \operatorname{Re}\left[G_{\chi}^{++}\left(x, x^{\prime}\right)\right]^{2}
$$

and

$$
\mathcal{N}_{2}\left(\mathbf{x}-\mathbf{x}^{\prime}, t-t^{\prime}\right)=\frac{\lambda^{2}}{6} \operatorname{Re}\left[G_{\phi}^{++}\left(x, x^{\prime}\right)\right]^{3}+2 g^{4} \operatorname{Re}\left[G_{\chi}^{++}\left(x, x^{\prime}\right)^{2} G_{\phi}^{++}\left(x, x^{\prime}\right)\right],
$$

and using again the properties of the field propagators in the CTP formalism, we notice that $\mathcal{N}_{i}$ are symmetrical in both space and time. Thus, their Fourier transforms in space and time, $\mathcal{F}\left[\mathcal{N}_{i}\left(x, x^{\prime}\right)\right]=\tilde{\mathcal{N}}_{i}(\mathbf{p}, \omega)$, are purely real.

From this point on, we will restrict ourselves to parameter values such that $\lambda=\mathcal{O}\left(g^{4}\right)$ and $g^{2} \ll 1$. In this situation we can drop the first term in the right-hand-side of both Eqs. (3.8) and (3.9) and likewise in the expressions for the kernels (3.2) and (3.3). Repeating the analysis with these terms included presents no specific difficulties, but it is not particularly illuminating.

In order to relate the dissipation and noise kernels, it is convenient to write the Feynman propagator $G^{++}\left(x, x^{\prime}\right)$ in real and imaginary parts as 28$]$

$$
G^{++}\left(x, x^{\prime}\right)=F\left(x, x^{\prime}\right)+\frac{1}{2} \rho\left(x, x^{\prime}\right) \operatorname{sgn}\left(t-t^{\prime}\right),
$$

where $F\left(x, x^{\prime}\right)$ and $\rho\left(x, x^{\prime}\right)$ are the anti-commutator and commutator (or the statistical and spectral functions) of two fields defined, respectively, by

$$
F\left(x, x^{\prime}\right)=\frac{i}{2}\left\langle\left\{\phi(x), \phi\left(x^{\prime}\right)\right\}\right\rangle=\frac{1}{2}\left[G^{<}\left(x, x^{\prime}\right)+G^{>}\left(x, x^{\prime}\right)\right],
$$

and

$$
\rho\left(x, x^{\prime}\right)=i\left\langle\left[\phi(x), \phi\left(x^{\prime}\right)\right]\right\rangle=\left[G^{>}\left(x, x^{\prime}\right)-G^{<}\left(x, x^{\prime}\right)\right] .
$$

For real fields, $F\left(x, x^{\prime}\right)$ and $\rho\left(x, x^{\prime}\right)$ are real and pure imaginary functions respectively, and have the symmetry properties $F\left(x, x^{\prime}\right)=F\left(x^{\prime}, x\right)$ and $\rho\left(x, x^{\prime}\right)=-\rho\left(x^{\prime}, x\right)$, which easily follow from the definitions (2.2) and (2.3). Note also that since $\rho\left(x, x^{\prime}\right)$ is an anti-symmetrical function, its Fourier transform, $\mathcal{F}\left[\rho\left(x, x^{\prime}\right)\right]=\tilde{\rho}$, is real.

In terms of (3.11) and (3.12), the noise kernel $\mathcal{N}_{1}$ becomes

$$
\begin{aligned}
\mathcal{N}_{1}\left(\mathbf{x}-\mathbf{x}^{\prime}, t-t^{\prime}\right) & =2 g^{4} \operatorname{Re}\left[G_{\chi}^{++}\left(x, x^{\prime}\right)\right]^{2} \\
& =2 g^{4}\left[F_{\chi}\left(\mathbf{x}-\mathbf{x}^{\prime}, t-t^{\prime}\right)^{2}+\frac{1}{4} \rho_{\chi}\left(\mathbf{x}-\mathbf{x}^{\prime}, t-t^{\prime}\right)^{2}\right],
\end{aligned}
$$


or in terms of Fourier transforms,

$$
\begin{aligned}
\mathcal{F}\left[\mathcal{N}_{1}\left(\mathbf{x}-\mathbf{x}^{\prime}, t-t^{\prime}\right)\right] & =\tilde{\mathcal{N}}_{1}(\mathbf{p}, \omega) \\
& =2 g^{4} \int \frac{d^{3} k}{(2 \pi)^{3}} \int_{-\infty}^{\infty} \frac{d \omega^{\prime}}{2 \pi}\left[\tilde{F}_{\chi}\left(\mathbf{k}, \omega^{\prime}\right) \tilde{F}_{\chi}\left(\mathbf{p}-\mathbf{k}, \omega-\omega^{\prime}\right)+\frac{1}{4} \tilde{\rho}_{\chi}\left(\mathbf{k}, \omega^{\prime}\right) \tilde{\rho}_{\chi}\left(\mathbf{p}-\mathbf{k}, \omega-\omega^{\prime}\right)\right]
\end{aligned}
$$

If we now use the property valid at equilibrium, coming from the Kubo-Martin-Schwinger relation for the propagators, $G^{>}(\mathbf{p}, \omega)=\exp (\beta \omega) G^{<}(\mathbf{p}, \omega)$, it follows, from the definition of the spectral density, that [29]

$$
\begin{aligned}
G^{>}(\mathbf{p}, \omega) & =[1+n(\omega)] \tilde{\rho}(\mathbf{p}, \omega), \\
G^{<}(\mathbf{p}, \omega) & =n(\omega) \tilde{\rho}(\mathbf{p}, \omega),
\end{aligned}
$$

where $n(\omega)$ is the Bose-Einstein distribution function,

$$
n(\omega)=\frac{1}{e^{\beta \omega}-1},
$$

and then we obtain for the Fourier transform of the statistical function, $\mathcal{F}\left[F\left(x, x^{\prime}\right)\right]=\tilde{F}(\mathbf{p}, \omega)$, the result

$$
\tilde{F}(\mathbf{p}, \omega)=\frac{1}{2}[1+2 n(\omega)] \tilde{\rho}(\mathbf{p}, \omega) .
$$

Using Eq. (3.17) and the identity

$$
1+\left[1+2 n\left(\omega^{\prime}\right)\right]\left[1+2 n\left(\omega-\omega^{\prime}\right)\right]=2\left(e^{\beta \omega}+1\right) n\left(\omega^{\prime}\right) n\left(\omega-\omega^{\prime}\right),
$$

we obtain for Eq. (3.14) the result

$$
\tilde{\mathcal{N}}_{1}(\mathbf{p}, \omega)=g^{4}\left(e^{\beta \omega}+1\right) \int \frac{d^{3} k}{(2 \pi)^{3}} \int_{-\infty}^{\infty} \frac{d \omega^{\prime}}{2 \pi} n\left(\omega^{\prime}\right) n\left(\omega-\omega^{\prime}\right) \tilde{\rho}_{\chi}\left(\mathbf{k}, \omega^{\prime}\right) \tilde{\rho}_{\chi}\left(\mathbf{p}-\mathbf{k}, \omega-\omega^{\prime}\right) .
$$

Analogously, for the kernel $\mathcal{C}_{1}\left(\mathbf{x}-\mathbf{x}^{\prime}, t-t^{\prime}\right)$, we find

$$
\begin{aligned}
\mathcal{C}_{1}\left(\mathbf{x}-\mathbf{x}^{\prime}, t-t^{\prime}\right) & =4 g^{4} \operatorname{Im}\left[G_{\chi}^{++}\left(x, x^{\prime}\right)\right]^{2} \operatorname{sgn}\left(t-t^{\prime}\right) \\
& =4 i g^{4} F_{\chi}\left(\mathbf{x}-\mathbf{x}^{\prime}, t-t^{\prime}\right) \rho_{\chi}\left(\mathbf{x}-\mathbf{x}^{\prime}, t-t^{\prime}\right),
\end{aligned}
$$

and expressing it in terms of transforms, after some algebra, we find

$$
\tilde{\mathcal{C}}_{1}(\mathbf{p}, \omega)=-2 i g^{4}\left(e^{\beta \omega}-1\right) \int \frac{d^{3} k}{(2 \pi)^{3}} \int_{-\infty}^{\infty} \frac{d \omega^{\prime}}{2 \pi} n\left(\omega^{\prime}\right) n\left(\omega-\omega^{\prime}\right) \tilde{\rho}_{\chi}\left(\mathbf{k}, \omega^{\prime}\right) \tilde{\rho}_{\chi}\left(\mathbf{p}-\mathbf{k}, \omega-\omega^{\prime}\right) .
$$

Using now Eq. (3.6) we finally find that the dissipation and noise kernel satisfy the generalized fluctuation-dissipation relation

$$
\tilde{\mathcal{N}}_{1}(\mathbf{p}, \omega)=2 \omega\left[n(\omega)+\frac{1}{2}\right] \tilde{\Gamma}_{1}(\mathbf{p}, \omega)
$$

Likewise, for the noise kernel $\mathcal{N}_{2}$ we find

$$
\begin{aligned}
\mathcal{N}_{2}\left(\mathbf{x}-\mathbf{x}^{\prime}, t-t^{\prime}\right) & =2 g^{4} \operatorname{Re}\left[G_{\chi}^{++}\left(x, x^{\prime}\right)^{2} G_{\phi}^{++}\left(x, x^{\prime}\right)\right] \\
& =2 g^{4}\left\{\left[F_{\chi}\left(\mathbf{x}-\mathbf{x}^{\prime}, t-t^{\prime}\right)^{2}+\frac{1}{4} \rho_{\chi}\left(\mathbf{x}-\mathbf{x}^{\prime}, t-t^{\prime}\right)^{2}\right] F_{\phi}\left(\mathbf{x}-\mathbf{x}^{\prime}, t-t^{\prime}\right)\right. \\
& \left.+\frac{1}{2} F_{\chi}\left(\mathbf{x}-\mathbf{x}^{\prime}, t-t^{\prime}\right) \rho_{\chi}\left(\mathbf{x}-\mathbf{x}^{\prime}, t-t^{\prime}\right) \rho_{\phi}\left(\mathbf{x}-\mathbf{x}^{\prime}, t-t^{\prime}\right)\right\}
\end{aligned}
$$


and in terms of Fourier transforms,

$$
\begin{aligned}
\tilde{\mathcal{N}}_{2}(\mathbf{p}, \omega) & =g^{4}\left(e^{\beta \omega}+1\right) \int \frac{d^{3} k}{(2 \pi)^{3}} \frac{d^{3} q}{(2 \pi)^{3}} \int_{-\infty}^{\infty} \frac{d \omega_{1}}{2 \pi} \frac{d \omega_{2}}{2 \pi} n\left(\omega_{1}\right) n\left(\omega_{2}\right) n\left(\omega-\omega_{1}-\omega_{2}\right) \\
& \times \tilde{\rho}_{\chi}\left(\mathbf{k}, \omega_{1}\right) \tilde{\rho}_{\chi}\left(\mathbf{q}, \omega_{2}\right) \tilde{\rho}_{\phi}\left(\mathbf{p}-\mathbf{k}-\mathbf{q}, \omega-\omega_{1}-\omega_{2}\right),
\end{aligned}
$$

while for the transform of the kernel $\mathcal{C}_{2}\left(\mathbf{x}-\mathbf{x}^{\prime}, t-t^{\prime}\right)$ we find

$$
\begin{aligned}
\tilde{\mathcal{C}}_{2}(\mathbf{p}, \omega) & =-2 i g^{4}\left(e^{\beta \omega}-1\right) \int \frac{d^{3} k}{(2 \pi)^{3}} \frac{d^{3} q}{(2 \pi)^{3}} \int_{-\infty}^{\infty} \frac{d \omega_{1}}{2 \pi} \frac{d \omega_{2}}{2 \pi} n\left(\omega_{1}\right) n\left(\omega_{2}\right) n\left(\omega-\omega_{1}-\omega_{2}\right) \\
& \times \tilde{\rho}_{\chi}\left(\mathbf{k}, \omega_{1}\right) \tilde{\rho}_{\chi}\left(\mathbf{q}, \omega_{2}\right) \tilde{\rho}_{\phi}\left(\mathbf{p}-\mathbf{k}-\mathbf{q}, \omega-\omega_{1}-\omega_{2}\right) .
\end{aligned}
$$

Upon using Eq. (3.6), we again find that the dissipation and noise kernels $\Gamma_{2}(\mathbf{p}, \omega)$ and $\tilde{\mathcal{N}}_{2}(\mathbf{p}, \omega)$ are also related in an identical way to Eq. (3.22).

These fluctuation-dissipation relations are actually generic to any product of two-point Green functions in the CTP formalism. To see this, begin by expressing the self-energy matrix elements in a similar way to the CTP propagators as

$$
\begin{aligned}
& \Sigma^{++}\left(x, x^{\prime}\right)=\Sigma^{>}\left(x, x^{\prime}\right) \theta\left(t-t^{\prime}\right)+\Sigma^{<}\left(x, x^{\prime}\right) \theta\left(t^{\prime}-t\right), \\
& \Sigma^{--}\left(x, x^{\prime}\right)=\Sigma^{>}\left(x, x^{\prime}\right) \theta\left(t^{\prime}-t\right)+\Sigma^{<}\left(x, x^{\prime}\right) \theta\left(t-t^{\prime}\right), \\
& \Sigma^{+-}\left(x, x^{\prime}\right)=-\Sigma^{<}\left(x, x^{\prime}\right), \\
& \Sigma^{-+}\left(x, x^{\prime}\right)=-\Sigma^{>}\left(x, x^{\prime}\right),
\end{aligned}
$$

From (3.26) the following property follows,

$$
\Sigma^{++}\left(x, x^{\prime}\right)+\Sigma^{+-}\left(x, x^{\prime}\right)+\Sigma^{-+}\left(x, x^{\prime}\right)+\Sigma^{--}\left(x, x^{\prime}\right)=0 .
$$

In addition, the self-energies in (3.26) are found in general to satisfy conditions similar to those for the propagators, such as

$$
\begin{aligned}
& \Sigma^{>}\left(x, x^{\prime}\right)=\Sigma^{<}\left(x^{\prime}, x\right) \\
& {\left[i \Sigma^{>(<)}\left(x, x^{\prime}\right)\right]^{\dagger}=i \Sigma^{<(>)}\left(x, x^{\prime}\right) .}
\end{aligned}
$$

The real and imaginary parts for the self-energy component $\Sigma^{++}(p)$ in the CTP formalism can be separated by introducing the commutator and anticommutator functions, analogous to the ones used to express the causal propagator Eq. (3.10),

$$
\Sigma^{++}\left(x, x^{\prime}\right)=\Sigma_{F}\left(x, x^{\prime}\right)+\frac{1}{2} \Sigma_{\rho}\left(x, x^{\prime}\right) \operatorname{sgn}\left(t-t^{\prime}\right)
$$

where

$$
\begin{aligned}
& \Sigma_{\rho}\left(x, x^{\prime}\right)=\left[\Sigma^{>}\left(x, x^{\prime}\right)-\Sigma^{<}\left(x, x^{\prime}\right)\right], \\
& \Sigma_{F}\left(x, x^{\prime}\right)=\frac{1}{2}\left[\Sigma^{>}\left(x, x^{\prime}\right)+\Sigma^{<}\left(x, x^{\prime}\right)\right],
\end{aligned}
$$

which are pure imaginary and real respectively. Turning to our earlier example, Eq. (2.5), we find that the dissipation kernels are related to $\Sigma_{\rho}$ and the noise kernels to $\Sigma_{F}$.

For the dressed propagators as defined in (2.4), we can express the transform of the spectral function, $\tilde{\rho}(\mathbf{p}, \omega)$, in the form

$$
\tilde{\rho}(\mathbf{p}, \omega)=i\left(\omega^{2}-\omega(\mathbf{p})^{2}+\frac{1}{2} \Sigma_{F}+\frac{1}{2} i \tilde{\Sigma}_{\rho}\right)^{-1}-i\left(\omega^{2}-\omega(\mathbf{p})^{2}+\frac{1}{2} \Sigma_{F}-\frac{1}{2} i \tilde{\Sigma}_{\rho}\right)^{-1}
$$


where $\omega(\mathbf{p})^{2}=|\mathbf{p}|^{2}+M^{2}$. The Breit-Wigner form of Eq. (3.31) suggests that we can introduce a generalized relaxation time $\tau(\mathbf{p}, \omega)$ of the particle state via the relation

$$
\Sigma_{\rho}(\mathbf{p}, \omega)=4 \omega(\mathbf{p}) \tau(\mathbf{p}, \omega)^{-1} .
$$

The equilibrium self-energies can be expressed in terms of $\Sigma_{\rho}$,

$$
\begin{aligned}
& \tilde{\Sigma}^{>}(\mathbf{p}, \omega)=[1+n(\omega)] \Sigma_{\rho}(\mathbf{p}, \omega), \\
& \tilde{\Sigma}^{<}(\mathbf{p}, \omega)=n(\omega) \Sigma_{\rho}(\mathbf{p}, \omega) .
\end{aligned}
$$

Using Eq. (3.33), we find a local fluctuation dissipation relationship between the Fourier transforms of $\Sigma_{F}$ and $\Sigma_{\rho}$,

$$
\tilde{\Sigma}_{F}(\mathbf{p}, \omega)=\frac{1}{2}[1+2 n(\omega)] \tilde{\Sigma}_{\rho}(\mathbf{p}, \omega)
$$

where we have defined $\mathcal{F}\left[\Sigma_{F}\left(x, x^{\prime}\right)\right]=\tilde{\Sigma}_{F}(\mathbf{p}, \omega)$ and $\mathcal{F}\left[\Sigma_{\rho}\left(x, x^{\prime}\right)\right]=\tilde{\Sigma}_{\rho}(\mathbf{p}, \omega)$.

\section{LOCAL LIMIT FOR THE NOISE KERNELS}

We now study the possible localization of the effective EOM for the background field. We first note that nonlinear equations of the form of Eq. (3.4) are impossible to solve analytically. They are also notoriously difficult to solve numerically, since they typically exhibit highly oscillating nonlocal kernels that can lead to errors which quickly build up and that are too hard to control, thus preventing any simple numerical attempt to solve these kind of equations. There are however different approaches that can be applied to analyze these equations. For instance, in those cases where the kernels exhibit a strong exponential damping in time, like in the analysis performed in Refs. 15, 16], a numerical approach to analyze the nonlocal equation of motion for the background field is possible and these analysis have shown that a local approximation for the kernels, and then for the equation of motion, is in very good agreement with the full numerical solution of the nonlocal equation. There are however cases where such a strong damping behavior for the kernels are not possible (see e.g. Ref. [17]) and we must resort to other alternative analysis to determine how good a local approximation is for the nonlocal equation. For example what we are going to show below is the nonlocal kernel can be analyzed and used to infer the validity of the local approximation, in particular determine a time scale for locality; all this can be accomplished without reverting to solving complicated equations of motion like Eq. (3.4). This is our objective here. Note that since both dissipation and noise kernels are related, if we find that the noise kernels can be localized, then the same must apply to the dissipation kernels in the effective EOM. We shall consider homogeneous background fields, for which the main issue will be the localization in time. Throughout this section we assume that the relaxation time of the environment is sufficiently short to be neglected.

First of all, we shall define what we mean by a localization of the noise kernels $\mathcal{N}$. The concept of localization depends on a particular context. For example, if we are interested in the origin of density fluctuations in the early universe we might be interested in localization of the noise kernel on timescales smaller than the Hubble time. We shall therefore introduce the concept of localization with respect to a particular timescale $\tau$.

We shall say that a function $f(t)$ is slowly varying on a timescale $\tau$ if the Fourier transform $\tilde{f}(\omega)$ satisfies

$$
\tilde{f}(\omega)=0 \text { for } \omega>2 \pi / \tau .
$$

A kernel function $\mathcal{K}(t)$ will be described as localized on a timescale $\tau$ with accuracy $1-\epsilon$ if the Fourier transform $\tilde{\mathcal{K}}(\omega)$ satisfies

$$
\left|\frac{\tilde{\mathcal{K}}(\omega)-\tilde{\mathcal{K}}(0)}{\tilde{\mathcal{K}}(0)}\right|<\epsilon \text { for } \omega<2 \pi / \tau .
$$

It follows that, for $f(t)$ slowly varying on a timescale $\tau$ and $\mathcal{K}(t)$ localized on a timescale $\tau$,

$$
\int_{-\infty}^{\infty} \mathcal{K}\left(t-t^{\prime}\right) f\left(t^{\prime}\right) d t^{\prime}=\tilde{\mathcal{K}}(0) f(t)+\mathcal{O}(\epsilon)
$$


which is a formal way of stating that $\mathcal{K}(t) \approx \tilde{\mathcal{K}}(0) \delta\left(t-t^{\prime}\right)$. Note that, if $\tilde{\mathcal{K}}(\omega)$ is analytic in $\omega$, then the kernel can be localized and the kernel admits a local derivative expansion. In general, the derivative expansion might not exist, even when the kernel is localized in the sense given above.

To help understand the motivation for the above definition, it may be useful to think of a Gaussian function $\exp \left(-t^{2} / \tau_{1 / 2}^{2}\right)$ with width $\tau_{1 / 2}$. The corresponding width of the Fourier transform is $2 \pi / \tau_{1 / 2}$. By the definition adopted above, on the scale $\tau_{1 / 2}$, we would say that the function is not fully localized but roughly speaking about half localized, thus localized with accuracy around 50\%. For the Gaussian to appear localized in t-space, one actually should look at it from a much bigger scale of say $10 \tau_{1 / 2}$, which means a much smaller region in $\omega$-space near the maximum part of the gaussian where it does not vary too much, and thus $\epsilon$ in Eq. (4.2) would be small.

As shown in the previous section, the kernels $\mathcal{C}_{j}$ are related to the dissipation kernels $\mathcal{D}_{j}$ by Eq. (3.1), which transformed using $\omega$ (we omit the spatial variable for homogeneous fields) become,

$$
\tilde{\mathcal{D}}_{j}(\omega)=\frac{i}{\omega} \tilde{\mathcal{C}}_{j}(\omega)=2 \tilde{\Gamma}_{j}(\omega)
$$

However, from the fluctuation-dissipation relation (3.22), we have that

$$
\tilde{\mathcal{N}}_{j}(\omega)=2 \omega\left[n(\omega)+\frac{1}{2}\right] \tilde{\Gamma}_{j}(\omega)
$$

If a local approximation for the noise two-point correlation function exists, i.e. $\mathcal{N}\left(t-t^{\prime}\right) \equiv N_{0} \delta\left(t-t^{\prime}\right)$, then the noise amplitude $N_{0}$ will satisfy

$$
N_{0}=\tilde{\mathcal{N}}(0)=2 T \tilde{\Gamma}(0),
$$

where we have used Eq. (4.5) and taken the limit as $\omega \rightarrow 0$. Note in particular from Eq. (4.6) that there is no $T=0$ local limit for the noise correlation function and, thus, no local limit for the dissipation terms in the effective EOM (3.4) at $T=0$.

Equation (4.6) is one of our main results. This simple relationship between the noise and dissipation has appeared previously as a high temperature limit in various applications, but our derivation is quite general and depends only on the localizability of the noise and dissipation. We shall describe in the next subsection how, and under what circumstances, the localization in time is realized.

The noise amplitude $\tilde{\mathcal{N}}(0)$ can be determined as follows. The inverse transform of the one-loop and two-loop noise kernels Eqs. (3.19) and (3.24) is defined by

$$
\mathcal{F}^{-1}\left[\tilde{\mathcal{N}}_{i}(\mathbf{p}, \omega)\right]=\mathcal{N}_{i}\left(\mathbf{x}-\mathbf{x}^{\prime}, t-t^{\prime}\right)=\int \frac{d^{3} p}{(2 \pi)^{3}} \int_{-\infty}^{+\infty} \frac{d \omega}{(2 \pi)} \exp \left[-i \mathbf{p} \cdot\left(\mathbf{x}-\mathbf{x}^{\prime}\right)+i \omega\left(t-t^{\prime}\right)\right] \tilde{\mathcal{N}}_{i}(\mathbf{p}, \omega) .
$$

For homogeneous fields, $\varphi_{c}(x) \equiv \varphi_{c}(t)$, all loop diagrams in the effective action carries zero external space momentum, $\mathbf{p}=0$, and then immediately follows from Eq. (4.7) that $\mathcal{N}_{1}\left(\mathbf{x}-\mathbf{x}^{\prime}, t-t^{\prime}\right)$ and $\mathcal{N}_{2}\left(\mathbf{x}-\mathbf{x}^{\prime}, t-t^{\prime}\right)$ are delta correlated in space $\left(\mathcal{N}_{i}\left(\mathbf{x}-\mathbf{x}^{\prime}, t-t^{\prime}\right) \sim \delta\left(\mathbf{x}-\mathbf{x}^{\prime}\right)\right)$. If also a local limit for the noise two-point correlations are valid, then the noise amplitudes become, from Eqs. (3.19) and (3.24), respectively

$$
\tilde{\mathcal{N}}_{1}(0)=2 g^{4} \int \frac{d^{3} k}{(2 \pi)^{3}} \int_{-\infty}^{+\infty} \frac{d \omega_{1}}{2 \pi} n\left(\omega_{1}\right)\left[1+n\left(\omega_{1}\right)\right] \tilde{\rho}_{\chi}^{2}\left(\mathbf{k}, \omega_{1}\right)
$$

while for the two-loop noise kernel,

$$
\begin{aligned}
\tilde{\mathcal{N}}_{2}(0) & =2 g^{4} \int \frac{d^{3} k}{(2 \pi)^{3}} \frac{d^{3} q}{(2 \pi)^{3}} \int_{-\infty}^{\infty} \frac{d \omega_{1}}{2 \pi} \frac{d \omega_{2}}{2 \pi} n\left(\omega_{1}\right) n\left(\omega_{2}\right)\left[1+n\left(\omega_{1}+\omega_{2}\right)\right] \\
& \times \tilde{\rho}_{\chi}\left(\mathbf{k}, \omega_{1}\right) \tilde{\rho}_{\chi}\left(\mathbf{q}, \omega_{2}\right) \tilde{\rho}_{\phi}\left(\mathbf{k}+\mathbf{q}, \omega_{1}+\omega_{2}\right) .
\end{aligned}
$$

From now on we will assume the simplest case of homogeneous fields and study the localization for the noise kernels in time. If such a local approximation exists for the noise kernels, then Eq. (4.6) suggests that the Fourier $(\omega)$ transform, $\tilde{\mathcal{N}}_{j}(\omega)$, should not differ much from the $\omega=0$ value, at least for some region in $\omega$ space, $-\omega_{\text {local }} \lesssim \omega \lesssim \omega_{\text {local }}$. We now try to find this region in $\omega$ space. 


\section{A. The one-loop noise kernel}

For the general case, which should be valid for both the low as well high temperature limits, we can use the full expression for the spectral density, Eq. (3.31), which in terms of the decay width $\tau_{i}^{-1}=-\operatorname{Im} \Sigma_{i} /\left[4 \omega_{i}(\mathbf{k})\right]$, can be written in the form $(i=\phi, \chi)$

$$
\tilde{\rho}_{i}(\mathbf{k}, \omega)=\frac{4 \omega_{i}(\mathbf{k}) \tau_{i}^{-1}(\mathbf{k}, \omega)}{\left[\omega^{2}-\omega_{i}^{2}(\mathbf{k})\right]^{2}+\left[2 \omega_{i}(\mathbf{k}) \tau_{i}^{-1}(\mathbf{k}, \omega)\right]^{2}}
$$

We will now study the localization of the one-loop noise kernel Eq. (3.8). Recall that here we are restricting our analysis to the parameter regime where $\lambda \ll g^{2}$, where the first term in Eq. (3.8) can be neglected compared to the second one. The second term will only involve the spectral density for the $\chi$ field. The decay width $\tau_{\chi}^{-1}$ receives contributions from both one and two $\sigma$ loop diagrams. Both results are well known (see e.g. Ref. 17] for the first one and Ref. [13] for the second). The single $\sigma$ loop diagram is $\mathcal{O}\left(h^{2}\right)$, while the two-loop diagram is $\mathcal{O}\left(g^{4}, h^{2}\right)$. Therefore, for parameter values such that $h \gg g^{2}$ (these parameter values were in fact the ones shown to be relevant for the dynamics studied in Refs. [13, 14, 15, 16]), the dominant contribution comes from the single loop. Thus, for the dominant decay channel we have that $(k \equiv|\mathbf{k}|)[17]$

$$
\tau_{\chi}^{-1}(\mathbf{k}, \omega) \simeq \frac{h^{2} \mathcal{M}^{2}}{16 \pi \omega_{\chi}(\mathbf{k})}[\theta(\omega-k)-\theta(-\omega-k)]+\frac{h^{2} \mathcal{M}^{2}}{8 \pi \omega_{\chi}(\mathbf{k})} \frac{T}{k} \ln \left[\frac{1-e^{-\beta|\omega+k| / 2}}{1-e^{-\beta|\omega-k| / 2}}\right],
$$

which is valid for very light $\sigma$ bath fields, $m_{\sigma} \approx 0$.

Equation (3.19) then becomes (for $p \equiv|\mathbf{p}|=0$ )

$$
\tilde{\mathcal{N}}_{1}(\omega) \simeq g^{4}\left(e^{\beta \omega}+1\right) \int \frac{d^{3} k}{(2 \pi)^{3}} \int_{-\infty}^{\infty} \frac{d \omega^{\prime}}{2 \pi} n\left(\omega^{\prime}\right) n\left(\omega-\omega^{\prime}\right) \tilde{\rho}_{\chi}\left(\mathbf{k}, \omega^{\prime}\right) \tilde{\rho}_{\chi}\left(\mathbf{k}, \omega-\omega^{\prime}\right)
$$

\section{The low temperature limit}

At low temperatures $T \ll m_{\chi}$, we can easily verify that the dominant contributions to the noise kernel Eq. (3.19) come from $k, \omega \ll m_{\chi}$. We can approximate the spectral function Eq. (3.31) for the $\chi$ field as

$$
\rho_{\chi} \simeq \frac{4}{m_{\chi}^{3}} \tau_{\chi}^{-1}
$$

with $\omega_{\chi}(\mathbf{k}) \sim m_{\chi}$ in Eq. (4.11). Thus, from Eqs. (3.8), (3.19) and (4.13), and for the same parameter values considered before, $h \gg f, g^{2}$ and $\lambda \ll g^{2}$, we obtain

$$
\tilde{\mathcal{N}}_{1}(\omega) \simeq \frac{16 g^{4}}{m_{\chi}^{6}}\left(e^{\beta \omega}+1\right) \int \frac{d^{3} k}{(2 \pi)^{3}} \int_{-\infty}^{\infty} \frac{d \omega^{\prime}}{2 \pi} n\left(\omega^{\prime}\right) n\left(\omega-\omega^{\prime}\right) \tau_{\chi}^{-1}\left(\mathbf{k}, \omega^{\prime}\right) \tau_{\chi}^{-1}\left(\mathbf{k}, \omega-\omega^{\prime}\right) .
$$

The dimensionful parameters can all be rescaled out of the integral to leave

$$
\tilde{\mathcal{N}}_{1}(\omega)=g^{4} h^{4} \frac{\mathcal{M}^{4} T^{4}}{m_{\chi}^{8}} \mathrm{~F}(h, \beta \omega) .
$$

In principle $\mathrm{F}$ only depends on $\beta \omega$, but in practice there is a breakdown of (4.13) at $\omega=k$ when the light field mass $m_{\sigma}=0$, resulting in the $h$ dependence.

The results of a numerical evaluation of the function $\mathrm{F}(h, \beta \omega)$ have been plotted in Fig. 1, which takes the temperature $T / m_{\chi}=0.01$ for the cases $h=0.1$ (solid) and 1.0 (dashed). Below this temperature, the numerical calculations find almost no change in $\mathrm{F}(h, \beta \omega)$, which is consistent with our parameterization. The best 3-parameter fit to the numerical curve at small values of $x=\omega / T$ is of the form 


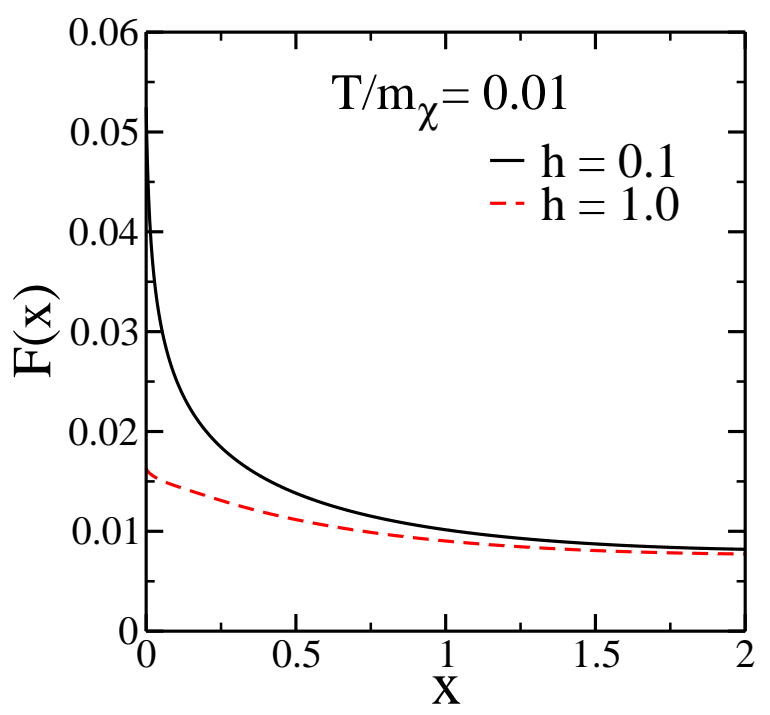

FIG. 1: The rescaled noise amplitude $\mathrm{F}(h, x)$, where $x=\omega / T$, at $T / m_{\chi}=0.01$ for $h=0.1$ (solid) and 1.0 (dashed). Below this temperature these curves do not change significantly.

$$
\mathrm{F}(h, x)=A(h)+B(h) x \ln (x)+C(h) x,
$$

where we find $A(0.1)=0.0458, B(0.1)=0.144$ and $C(0.1)=0.126$ and $A(1.0)=0.0161, B(1.0)=0.0068$ and $C(1.0)=-0.0004$. These numerical results provide convincing evidence that the noise kernel can be localized in the sense described earlier. For example, we can say for $h=0.1$ that the noise kernel is localized on a timescale $1600 T^{-1}$ with $95 \%$ accuracy and for $h=1.0$ the noise kernel is localized on a timescale $100 T^{-1}$ with $95 \%$ accuracy. Note that, although this may be a large timescale compared to the scale of particle interactions, it may still be a small timescale compared to the timescale of a cosmological application.

It is interesting that the numerical evidence supports the conclusion that the noise kernel is not analytic in $\omega$. The logarithmic terms in $\omega$ are related to subleading non-local effects. This also has an important implication for the dissipation terms. The dissipation kernels $\mathcal{C}_{\chi}$ and $\mathcal{D}_{\chi}$ are related to the noise kernel by the fluctuation-dissipation relation Eq. (4.5), and in the low temperature limit,

$$
\tilde{\mathcal{C}}_{\chi}=-i \omega \tilde{\mathcal{D}}_{\chi}=-2 i \beta \omega \tilde{\mathcal{N}}
$$

Therefore the dissipation terms can have a local analytic expansion at leading order to give a $\dot{\varphi}$ term, but beyond that there is not a local analytic expansion but rather terms of the form

$$
\ln \left(\frac{\partial}{\partial t}\right) \frac{\partial^{2}}{\partial t^{2}} \varphi
$$

For a definition of the logarithmic derivative, see Ref. [30].

\section{The high temperature limit}

For high temperature $T \gg m_{\chi}$ and $T \gg \omega$, the specral function $\tilde{\rho}_{\chi}$ is concentrated in the range of values of $\omega^{\prime}$ close to $\pm \omega(\mathbf{p})$, where

$$
\omega_{\chi}(\mathbf{k})=\left(k^{2}+m_{\chi}^{2}\right)^{1 / 2} .
$$




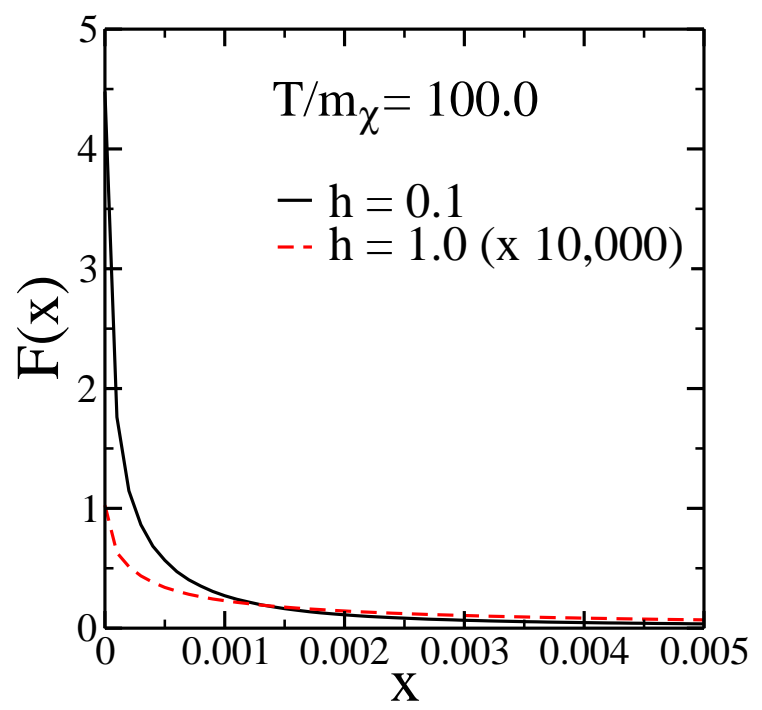

FIG. 2: The rescaled noise amplitude $\mathrm{F}(h, x)$, where $x=\omega / T$, at $T / m_{\chi}=100.0$ for $h=0.1$ (solid) and 1.0 (dashed, increase by a factor 10,000$)$.

We take the distribution functions out of the energy integral,

$$
\tilde{\mathcal{N}}_{1}(\omega) \approx-g^{4}\left(e^{\beta \omega}+1\right) \int \frac{d^{3} k}{(2 \pi)^{3}} n\left(\omega_{\chi}(\mathbf{k})\right)\left[1+n\left(\omega_{\chi}(\mathbf{k})\right)\right] \int_{-\infty}^{\infty} \frac{d \omega^{\prime}}{2 \pi} \tilde{\rho}_{\chi}\left(\mathbf{k}, \omega^{\prime}\right) \tilde{\rho}_{\chi}\left(\mathbf{k}, \omega-\omega^{\prime}\right)
$$

The spectral function is given by Eq. (4.10), with $\tau_{\chi}(k) \equiv \tau_{\chi}\left(\mathbf{k}, \omega_{\chi}(\mathbf{k})\right)$. After the $\omega^{\prime}$ integration,

$$
\tilde{\mathcal{N}}_{1}(\omega) \approx g^{4} \int \frac{d^{3} k}{(2 \pi)^{3}} \frac{4 \tau_{\chi}(k)}{4+\tau_{\chi}(k)^{2} \omega^{2}} \frac{n\left(\omega_{\chi}(\mathbf{k})\right)\left[1+n\left(\omega_{\chi}(\mathbf{k})\right)\right]}{\omega_{\chi}(\mathbf{k})^{2}}
$$

Note that this result for the noise kernel is consistent with the earlier relationship $\tilde{\mathcal{N}}_{1}(0)=2 T \tilde{\Gamma}(0)$, where $\tilde{\Gamma}(0)$ is the high temperature dissipation coefficient first calculated by Hosoya and Sakagami [7].

The noise kernel is localized in the sense which we defined earlier. For a rough estimate, we introduce the relaxation timescale $\tau_{\chi}$ at $k=T$ and take $\tau_{\chi}$ out of the $k$ integral. The noise kernel is then localized at the timescale $\sim 30 \tau_{\chi}$ with accuracy $99 \%$. Alternatively, we can say in less formal terms that the noise kernel appears localized for physics on timescales larger than the relaxation time. A numerical calculation of the noise amplitude is shown in Fig. 2 and it is consistent with these analytic estimates of the localization scale.

\section{B. The two-loop noise kernel}

For the two-loop noise kernel, the expression analogous to Eq. (4.12), from Eq. (3.24), is

$$
\begin{aligned}
\tilde{\mathcal{N}}_{2}(\omega) & =g^{4}\left(e^{\beta \omega}+1\right) \int \frac{d^{3} k}{(2 \pi)^{3}} \frac{d^{3} q}{(2 \pi)^{3}} \int_{-\infty}^{\infty} \frac{d \omega_{1}}{2 \pi} \frac{d \omega_{2}}{2 \pi} n\left(\omega_{1}\right) n\left(\omega_{2}\right) n\left(\omega-\omega_{1}-\omega_{2}\right) \\
& \times \tilde{\rho}_{\chi}\left(\mathbf{k}, \omega_{1}\right) \tilde{\rho}_{\chi}\left(\mathbf{q}, \omega_{2}\right) \tilde{\rho}_{\phi}\left(\mathbf{k}+\mathbf{q}, \omega-\omega_{1}-\omega_{2}\right)
\end{aligned}
$$

where $\tilde{\rho}_{\chi}$ is given by Eq. (4.10) with decay width given by Eq. (4.11), and $\tilde{\rho}_{\phi}$ is given by an equivalent expression with $\tau_{\phi}$ in place of $\tau_{\chi}$. For the range of parameters discussed above, the dominant decay channel for $\phi$ in the thermal bath is the two-loop channel $\phi \rightarrow \phi \chi \chi$. The explicit expression for this decay term (and for any of the two-loop terms in general) is a very complicated one and there is no simple result available for it, unfortunately. An explicit expression 
in terms of momenta integrals was derived in [13], for the on-shell case $\omega=\omega(\mathbf{p})$ only. However, we note that for the dominant decay channel $\phi \rightarrow \phi \chi \chi, \tau_{\phi}^{-1} \sim \mathcal{O}\left(g^{4}\right)$, and since in Eq. (4.22) we already have a $g^{4}$ factor and in each of the $\tilde{\rho}_{\chi}$ terms we have an extra factor of $h^{2}$ (from Eq. 4.11) ), then $\tilde{\mathcal{N}}_{2} \sim \mathcal{O}\left(g^{8} h^{4}\right)$ and it is subleading compared to the one-loop kernel $\tilde{\mathcal{N}}_{1}$. Because of this, there is no need to proceed further with an analysis of the two-loop term here.

\section{CONCLUSIONS}

The effective (microscopic) equations of motion of systems coupled to an environment in the form of a heat bath typically include nonlocal dissipation and noise (stochastic) kernels. These terms are generally complicated and need to be dealt with somehow for practical purposes for studying the dynamical evolution of these systems. The same is true for the evolution of quantum fields in a medium. In this paper we have studied and approached the problem of localization of the nonlocal kernels in the context of the dynamics of a scalar background field coupled to an environment made of other scalar fields. We have paid special attention to a particular model where dissipation emerges as a consequence of a two-stage decay mechanism where a scalar field decays into a light one through a catalyst heavy field. This model was identified in Refs. [15, 16] and can emerge naturally in the context of Grand Unified and Supersymmetric theories, with applications in several contexts, like inflationary cosmology and particle physics dynamics in general.

For this model, we have studied in this paper the nonlocal dissipation and noise kernels that emerge in general in the microscopic derivation of the effective equations of motion for quantum fields. From this we have derived a fluctuation-dissipation theorem and determined the appropriate conditions for when the kernel terms can be well approximated by a local form.

We should point out that small differences between the local and nonlocal kernels over large evolution timescales can still manifest themselves as a secular drift. This, if the scalar field is oscillating, can lead to a phase shift that might accumulate, but does not affect the important physical quantities like the rate of dissipation of energy. If the dissipation terms dominate over the other kinetic terms, then the overdamped solution emerges and a small correction to the equations is equivalent to a shift in the origin of time, which has no physical significance.

For a scalar field with a expectation value $\varphi_{c}$ we have found that, in certain situations when the local form is possible, the effective equation of motion reduces to

$$
\left[\square+M_{\phi}^{2}\right] \varphi_{c}(x)+2 \Gamma(\varphi, T) \varphi_{c}^{2}(x) \dot{\varphi}_{c}(x)+\frac{\lambda}{3 !} \varphi_{c}^{3}(x)=\varphi_{c}(x) \xi(x),
$$

where the stochastic noise source has a correlation function

$$
\left\langle\xi(x) \xi\left(x^{\prime}\right)\right\rangle=2 \Gamma(\varphi, T) T \delta\left(x-x^{\prime}\right) .
$$

The situations which we have examined have been for approximately homogeneous fields coupled to the heat bath through a catalyst field with coupling $g$ and weak self-coupling $\lambda=O\left(g^{2}\right)$ :

1. When the catalyst mass is large compared to the temperature and the scalar field is slowly varying on a timescale $C T^{-1}$, where $C$ is a constant which was determined numerically.

2. When the catalyst mass is small compared to the temperature and the scalar field is slowly varying on the relaxation timescale of the catalyst field.

In case 1, we have also given numerical evidence that the dissipative term is localized on a specified timescale but beyond leading order it possesses no analytic expansion with respect to the time coordinate, since beyond this order it is dominated by logarithmic terms.

Finally, we would like to point out a similar model to the one we analyzed here that was studied by the authors in Ref. 31]. In that case, they have studied the process of how the homogeneous field $\phi$ can produce fluctuations of $\chi$ through the process of parametric resonance. In that case, oscillations of the scalar field $\phi$ lead to the growth of the effective mass of $\chi$, which can then cause it to eventually decay into excitations of $\sigma$, even if $m_{\sigma}$ is much greater than the vacuum mass of $\phi$ or $\chi$. Though they analyze a different dynamical regime for the scalar field $\phi$, we think that this process of energy transfer from the field $\phi$ to $\sigma$, through parametric resonance, is a nice counterpart to the quantum process we have studied here. 


\section{Acknowledgments}

This work was partially supported by a U.K. Particle Physics and Astronomy Research Council (PPARC) Visiting Researcher Grant. In addition A.B. is partially supported by PPARC and R.O.R. is partially supported by Conselho Nacional de Desenvolvimento Científico e Tecnológico (CNPq - Brasil) and Fundação de Amparo à Pesquisa do Estado do Rio de Janeiro (FAPERJ).

[1] I. G. Moss, Phys. Lett. B154, 120 (1985).

[2] A. Berera, Phys. Rev. Lett. 75, 3218 (1995); Phys. Rev. D 54, 2519 (1996).

[3] A. N. Taylor, and A. Berera, Phys. Rev. D 62, 083517 (2000).

[4] L. M. H. Hall, I. G. Moss, and A. Berera, Phys. Rev. D 69, 083525 (2004); Phys. Lett. B 589, 1 (2004).

[5] Z. Xu and C. Greiner, Phys. Rev. D62, 036012 (2000).

[6] D. Boyanovsky and H. J. de Vega, hep-ph/9909372.

[7] A. Hosoya and M. Sakagami, Phys. Rev. D29, 2228 (1984).

[8] M. Morikawa and M. Sasaki, Phys. Lett. B165, 59 (1985); M. Morikawa, Phys. Rev. D33, 3607 (1986).

[9] A. Ringwald, Ann. Phys. (N.Y.) 177, 129 (1987).

[10] D. Boyanovsky and H. J. de Vega, Phys. Rev. D47, 2343 (1993).

[11] M. Gleiser and R. O. Ramos, Phys. Rev. D50, 2441 (1994).

[12] F. Cooper, S. Habib, Y. Kluger, E. Mottola, J. P. Paz and P. R. Anderson, Phys. Rev. D50, 2848 (1994).

[13] A. Berera, M. Gleiser and R. O. Ramos, Phys. Rev. D58, 123508 (1998).

[14] A. Berera, M. Gleiser and R. O. Ramos, Phys. Rev. Lett. 83, 264 (1999).

[15] A. Berera and R. O. Ramos, Phys. Rev. D 63, 103509 (2001).

[16] A. Berera and R. O. Ramos, Phys. Rev. D 71, 023513 (2005).

[17] I. G. Moss and C. Xiong, hep-ph/0603266.

[18] M. Bastero-Gil and A. Berera, Phys. Rev. D 76, 043515 (2007).

[19] J. Schwinger, Jour. Math. Phys. (N.Y.) 2, 407 (1961); P. M. Bakshi and K. T. Mahanthappa, Jour. Math. Phys. (N.Y.) 4, 1 (1963); 4, 12 (1963); L. V. Keldysh, Sov. Phys. JETP 20, 1018 (1964); A. Niemi and G. Semenoff, Ann. Phys. (NY) 152, 105 (1984); Nucl. Phys. B230, 181 (1984).

[20] T. Koide, G. Krein and R. O. Ramos, Phys. Lett. B636, 96 (2006).

[21] A. Das and M. Hott, Phys. Rev. D50, 6655 (1994).

[22] I. G. Moss, Nucl. Phys. B631, 500 (2002).

[23] G. Aarts and A. Tranberg, Phys. Lett. B650, 65 (2007).

[24] K. Chou, Z. Su, B. Hao and L. Yu, Phys. Rep. 118, 1 (1985).

[25] An accessible introduction to the real-time formalism can be found in R. Rivers, Path Integral Methods in Quantum Field Theory, Cambridge University Press, (Cambridge 1987).

[26] N. P. Landsman and Ch. G. van Weert, Phys. Rep. 145, 141 (1987).

[27] J. Yokoyama, Phys. Rev. D70, 103511 (2004).

[28] G. Aarts and J. Berges, Phys. Rev. D 64, 105010 (2001).

[29] M. Le Bellac, Thermal Field Theory (Cambridge University Press, Cambridge, 1996).

[30] I. G. Moss and J. P. Norman, Phys. Rev. D 70, 103506 (2004).

[31] G. Felder, L. Kofman and A. Linde, Phys. Rev. D 59, 123523 (1999). 